\title{
Politique
}

\section{La France entre le socialisme et l'austérité}

\section{Charles Larochelle}

Numéro 4, automne 1983

Crise et changements idéologiques

URI : https://id.erudit.org/iderudit/041003ar

DOI : https://doi.org/10.7202/041003ar

Aller au sommaire du numéro

Éditeur(s)

Société québécoise de science politique

ISSN

0711-608X (imprimé)

1918-6584 (numérique)

Découvrir la revue

Citer cet article

Larochelle, C. (1983). La France entre le socialisme et l'austérité. Politique, (4), 105-127. https://doi.org/10.7202/041003ar d'utilisation que vous pouvez consulter en ligne.

https://apropos.erudit.org/fr/usagers/politique-dutilisation/ 


\title{
La France entre \\ le socialisme et l'austérité
}

\author{
Charles LAROCHELLE \\ Université de Montréal
}

L'élection de François Mitterrand à la présidence de la République le 10 mai 1981, aussitôt suivie par celle d'une majorité socialiste, devait être le prélude de changements importants au sein de la société française. Vingt-quatre mois plus tard, qu'en est-il des transformations amorcées ou opérées par le nouveau régime? Des espoirs d'une gestion différente de sortie de la crise économique? C'est à ces questions que je tenterai d'apporter un éclairage, nécessairement partiel à ce stade-ci de l'expérience en cours; une expérience dont la trajectoire ne peut qu'être riche d'enseignements pour ceux qui s'interrogent sur les potentialités et les limites que recèle l'accession de la gauche au pouvoir dans un pays comme la France dans la conjoncture actuelle.

Précisons tout de suite que ce bilan concernera essentiellement les aspects économiques et sociaux de l'action gouvernementale, et qu'en sera donc écarté tout le champ de la politique étrangère. Il s'articulera autour de deux axes: il s'agira d'abord de dégager les grandes lignes de la politique économique d'ensemble, à partir des deux phases qui l'ont caractérisée jusqu’à maintenant; suivra une présentation des réformes majeures en- 
treprises sur le plan des structures (nationalisations, décentralisation, droits nouveaux des travailleurs) et de certaines des questions que soulève leur application. Je verrai brièvement, en guise de conclusion, les répercussions au sein de la population et plus spécifiquement de la gauche française de la politique menée par les socialistes.

\section{La gestion de la crise:}

a) Un an de politique expansionniste ${ }^{1}$

Aussitôt en selle, le gouvernement socialiste fait le pari d'une politique résolument expansionniste pour combattre la crise économique, option qui se présente comme une alternative à la gestion monétariste pratiquée dans la plupart des pays occidentaux. L'objectif visé est d'obtenir, par le biais d'une relance de la consommation, une reprise de l'investissement et de l'activité économique susceptible de déboucher sur la création d'emplois; celle-ci étant considérée prioritaire à la maîtrise de l'inflation. À l'arsenal keynésien traditionnel-revalorisation des prestations sociales et augmentation des dépenses publiques vont ainsi venir se greffer certaines mesures plus originales visant au partage du travail.

En ce qui concerne d'abord les prestations sociales le minimum vieillesse a été relevé de $20 \%$ le er juillet 1981 et de $18 \%$ le $1^{\text {er }}$ janvier 1982; les allocations familiales l'ont été de $25 \%$ le $1^{\text {er }}$ juillet 1981 et à nouveau de $25 \%$ pour deux enfants en février 1982; l'allocation logement a été deux fois augmentée, d'un total de $50 \%$. Au-delà de leurs effets sur la consommation, ces mesures correspondaient bien entendu au souci de

1. Pour un exposé assez détaillé des mesures adoptées pendant cette période sur le plan économique et social, voir Bela Balassa, "Une année de politique économique en France", Commentaire, no 19 , automne 1982, 415-429. 
réduire les inégalités et de soulager les plus démunis des effets de la crise.

Sur le plan de la redistribution, d'autres mesures ont été adoptées notamment au chapitre de la fiscalité. Outre la chasse aux fraudeurs, dont les résultats s'avèrent plutôt limités, les seuils de non-imposition ont été relevés, soustrayant deux millions de ménages modestes à l'impôt direct, une tranche d'imposition à $65 \%$ a été appliquée sur les gros revenus, et un impôt sur les grandes fortunes a été créé. Mais celui-ci, accueilli par un flot de protestations, a rapidement pris des proportions plus modestes, suite à l'exonération des ouvres d'art, des droits de la propriété littéraire et de la propriété industrielle. On ne peut donc pas parler de véritable réforme fiscale, d'autant plus que rien n'a été fait pour corriger le vice fondamental de la fiscalité française, à savoir la part prépondérante de l'impôt sur la consommation. La taxe à la valeur ajoutée (TVA) rapporte en effet près de deux fois le produit de l'impôt sur le revenu, alors qu'elle frappe davantage en valeur relative les classes populaires pour qui la consommation occupe une plus grande part du budget.

En matière de salaires, le gouvernement a écarté pendant cette première année toute norme législative ou réglementaire, à l'exception du SMIC (salaire minimum) qui a connu une forte hausse de $25 \%$ de mai 1981 à mai 1982. Pour le reste, le gouvernement s'en est remis à l'effet d'affichage du secteur public, et de façon générale les salaires ont progressé à un rythme au moins équivalent à celui du coût de la vie.

Par ailleurs, tout un train de mesures ont été adoptées qui tentent d'associer partage du travail et avancée sociale. Une première réduction de la durée de travail hebdomadaire, de 40 à 39 heures, sans diminution de salaire, a été décrétée; l'objectif initialement proclamé étant de ramener cette durée à 35 heures en 1985. À quoi il faut ajouter l'octroi d'une cinquième semaine 
de congés payés, la possibilité d'accéder au temps partiel plus librement et avec les mêmes garanties qu'un travail à temps plein, l'amélioration de la protection des intérimaires, la limitation des heures supplémentaires, et surtout l'institution des «contrats de solidarité». ${ }^{2} \mathrm{Par}$ ce dispositif, des aides publiques sont octroyées aux entreprises qui signent avec leurs employés un accord prévoyant soit une diminution de la durée de travail, soit la pré-retraite progressive à cinquante-cinq ans, avec en contrepartie l'embauche de nouveaux travailleurs.

Devant le problème du chômage des jeunes, un programme de 100000 stages de formation professionnelle a été mis à la disposition de tous ceux, âgés de seize à dix-huit ans, qui abandonnent leurs études dès la fin de la scolarité obligatoire sans diplôme. Ce programme a par la suite été élargi aux jeunes de 18 à 25 ans. Enfin, un effort considérable a été accordé à la création de nouveaux emplois dans les secteurs publics, les prévisions s'élevant à 55000 pour 1982. Selon le ministre de la Santé, les effectifs du personnel hospitalier auraient progressé à eux seuls de 12000 pendant les six premiers mois de son ministère. Les freins qui avaient commencé à être appliqués sous le précédent gouvernement à l'augmentation des dépenses de santé ont été levés et celles-ci ont enregistré un bond considérable, à l'instar de l'ensemble des dépenses publiques dont la croissance s'est chiffrée à $21 \%$ en 1981.

Quels ont été les résultats de cette politique du point de vue macro-économique? Il apparaît d'abord que la relance de la demande intérieure, stimulée par la hausse des salaires et des transferts publics, n'a pas provoqué une reprise significative de l'investissement privé et de l'activité économique. Les entreprises industrielles n'ont pas su ou pas pu, selon les points de vue,

2. Plus de 25000 contrats de solidarité ont été signés en 1982, concernant environ trois cent mille bénéficiaires potentiels d'une pré-retraite-démission. Les départs effectifs en 1982 dans le cadre de ces contrats sont de l'ordre de quatre vingt mille. 
répondre à ce surcroît de demande, ce qui allait avoir des conséquences déterminantes. On observe un tassement de la progression du chômage, encore assez modeste compte tenu des mesures adoptées. De plus, avec l'augmentation des investissements et des emplois dans le secteur public, le déficit budgétaire est passé, en dépit du relèvement de $1 \%$ du taux de TVA, de 30 milliards de francs en 1980 à 81 milliards en 1981. Les déficits de la Sécurité sociale et de l'UNEDIC (organisme conjoint syndical-patronal chargé de la gestion du système d'assurancechômage) se sont aussi creusés malgré l'augmentation des prélevements sur les employeurs et les salariés.

Les mesures expansionnistes adoptées par le gouvernement et la hausse des traitements et des salaires ont provoqué une accélération de l'inflation - qui a atteint un taux de $13.9 \%$ entre avril 1981 et avril 1982 - contrastant avec sa chute dans les autres grands pays industriels. Cette augmentation de la différence entre le taux d'inflation en France et celui de ses principaux partenaires commerciaux, jointe au fait que le franc se trouvait déjà surévalué au moment de l'arrivée des socialistes au pouvoir, a rendu nécessaire une première dévaluation de la monnaie en octobre 1981.

Mais ce qui est plus grave, la relance de la consommation s'est principalement traduite par une augmentation des importations et la détérioration de la balance commerciale. Cette fameuse contrainte extérieure, selon laquelle une politique de croissance et de déficit budgétaire a des répercussions négatives sur le plan des échanges extérieurs, a conduit le gouvernement à reviser ses choix de politique économique. L'intégration de la France dans une économie mondiale en récession, réalité dont on avait d'abord plus ou moins feint d'ignorer les conséquences, allair devenir une variable-clé pour la détermination de la conduite à suivre. Le déclin de la compétitivité des produits français, provoqué par l'inflation, et la croissance inquiétante du 
déficit du commerce extérieur devaient d'ailleurs inciter le gouvernement à opérer une seconde dévaluation du franc, de $10 \%$ par rapport au mark allemand, en juin 1982. Ce sera aussi l'occasion d'un changement de cap dans la gestion de la crise, une gestion qui privilégie désormais le rétablissement des grands équilibres économiques.

\section{b) De la rigueur à l'austérité}

La deuxième année de gouvernement socialiste se caractérise par un net retournement de la politique économique, qui va s'opérer en deux étapes. Il y aura d'abord, en accompagnement de la dévaluation de juin 1982, l'instauration d'un blocage des prix et des salaires pour une durée de quatre mois. Puis, pour éviter tout dérapage à la sortie de la période de gel, le gouvernement a pris certaines mesures visant à la modération des prix (conclusion d'accords branche par branche, limitation des hausses de tarifs des services publics) et des salaires. À cet égard, les hausses consenties ne devaient pas excéder $10 \%$ pour l'année 1982 et $8 \%$ pour 1983 , ces taux correspondant aux objectifs poursuivis en matière d'inflation. Les employés de la Fonction publique, et à leur suite ceux de certains services publics, se sont néanmoins vus concéder une formule d'indexation des salaires au coût de la vie ("clause de sauvegarde») si l'augmentation de celui-ci devait s'avérer supérieure aux prévisions. Le SMIC a échappé à la règle des $10 \%$ en gagnant environ $4 \%$ en pouvoir d'achat pour l'année 1982.

Si le niveau de vie des Français, mesuré en terme de revenu disponible des ménages, a progressé globalement de $2.2 \%$ en 1982, c'est en fait essentiellement grâce à la majoration des prestations sociales. Un frein a néanmoins été appliqué à la croissance de celles-ci comme en témoignent le renvoi partiel à une date ultérieure de l'augmentation des allocations familiales et de l'allocation-logement qui aurait dû entret en vigueur le $1^{\mathrm{er}}$ 
juillet 1982, et la réduction des indemnités de chômage. D'autres mesures ont été prises pour freiner l'augmentation des dépenses de l'État, qui a tout de même atteint $17.5 \%$ en 1982 et que le budget de 1983 prévoit de limiter à environ $12 \%$.

Le gouvernement s'est aussi engagé à stabiliser les charges sociales $^{3}$ des entreprises, de façon à ne pas grever davantage leurs marges bénéficiaires. En contrepartie des augmentations antérieures de ces charges, il a diminué la taxe professionnelle que doivent acquitter les entreprises. Il a aussi proposé, pour contrebalancer le relèvement des cotisations des employeurs à l'UNEDIC, de procéder à la fiscalisation des allocations familiales. En fait, la seconde phase inaugurée en juin se caractérise par une volonté assez nette de rapprochement avec le patronat. Cette attitude plus conciliante et les mesures d'aide aux entreprises sont justifiées par la nécessité d'obtenir le concours du secteur privé pour assurer la réussite de la politique économique.

Outre la modération des revendications salariales, la politique de rigueur s'est incarnée notamment dans la décision de reporter à 1984 toute nouvelle réduction obligatoire de la durée hebdomadaire de travail, laissant cependant aux partenaires sociaux le loisir de négocier certains aménagements. Il est donc à peu près assuré que l'objectif de 35 heures fixé pour 1985 ne sera pas atteint. Il faut souligner en contrepartie les résultats significatifs enregistrés dans la lutte contre le chômage. Alors que le nombre des sans-emplois progressait à un rythme accéléré dans les autres pays membres de l'OCDE en 1982, il n'a connu qu'une hausse de $5.8 \%$ en France - comparativement à $23.4 \%$ en 1981 - et le taux de chômage s'y est stabilisé à $9.5 \%$ depuis juin 1982. Comme on l'a souligné, cette victoire relative résulte des dispositions adoptées en faveur du partage du travail

3. Si les charges sociales supportées par les entreprises françaises sont plus élevées que dans la plupart des pays industrialisés, il faut souligner que l'importance de ce salaire indirect y est compensée par la faiblesse relative du salaire direct. 
et de la formation professionnelle des jeunes plutôt que d'une relance de l'industrie.

L'inflation a quant à elle vu son taux ramené, grâce à la politique de blocage, sous la barre des $10 \%$ en 1982. Cela n'a cependant pas suffi à résorber l'écart entre la France et ses principaux concurrents qui pendant ce temps réussissaient à contenir leur inflation à moins de $5 \%$. Pour les raisons que nous avons évoquées précédemment, le déficit commercial a pris une ampleur considérable, s'élevant à 93 milliards de francs en 1982, comparativement à 60 milliards l'année précédente, et le déficit des administrations a grimpé à 100 milliards. La France a donc dû s'endetter lourdement pour combler ces déficits et soutenir sa monnaie.

Face à la détérioration de la situation au chapitre de l'inflation et de la balance extérieure pendant les premiers mois de l'année, le gouvernement a finalement procédé, après les élections municipales et contrairement à ses engagements, à une troisième dévaluation. Cette fois le pouvoir d'achat ne sera pas épargné ${ }^{4}$. C'est un véritable plan d'austérité qui a été mis en place en mars 1983 pour freiner la consommation populaire au profit d'un rééquilibrage des échanges extérieurs. On entend en fait réaliser une ponction d'environ $2 \%$ sur la demande intérieure soit 65 milliards de francs. Les moyens sont variés: emprunt obligatoire représentant $10 \%$ de l'impôt payé en 1982 à compter de 5000 francs (le tiers des contribuables sont visés), prélèvement de $1 \%$ sur les revenus imposables de 1982 (touchant cette fois les deux tiers des contribuables) pour renflouer les coffres de la Sécurité sociale, instauration d'un forfait hospitalier et d'une vignette sur les alcools et tabacs ${ }^{5}$, compression

4. L'INSEE prévoyait en avril dernier une baisse d'environ $1.3 \%$ du revenu disponible des ménages en 1983.

5. Ces deux mesures avaient été annoncées dès septembre 1982, mais aucune date d'application n'avait été prévue, leur entrée en vigueur étant fonction de l'évolution de la situation financière de la Sécurité sociale. 
des dépenses publiques de 15 milliards de francs ${ }^{6}$, réduction des dépenses et majoration des tarifs des entreprises publiques du secteur de l'énergie et du transport, limitatioñ des dépenses touristiques à l'étranger ${ }^{7}$. Les catégories les plus modestes n'étant directement affectées que par la hausse des prix des services publics, il appert que ce sont les classes plus aisées qui devraient assumer l'essentiel de la réduction de la consommation, à moins qu'elles ne puisent dans leur épargne pour compenser la diminution de leur pouvoir d'achat.

S'il est encore trop tôt pour connaître les effets réels de cette batterie de mesures, il est à peu près assuré que les objectifs fixés pour 1983 en matière d'inflation $(8 \%)$ et de déficit commercial ( 45 milliards de francs) ne seront pas atteints. De plus, il est prévisible que le ralentissement de la croissance provoque une augmentation du nombre de chômeurs ${ }^{8}$. Si l'emploi occupe toujours le premier rang dans le discours gouvernemental, le retournement opéré dans le mode de gestion de la crise indique bien où se situent maintenant les priorités. La question qui se pose est évidemment de savoir s'il était possible de faire autrement. Dans la mesure où la France entend rester ouverte sur l'extérieur, il est impérieux qu'elle se soucie de la compétitivité de ses produits et par conséquent tente de combler le différentiel d'inflation par rapport à ses partenaires. Dans cette perspective, il devenait intenable de continuer une politique s'inscrivant à contre-courant de l'environnement international. Face à cette contrainte extérieure, une autre stratégie envisageable aurait résidé dans une sorte de repli protectionniste: sortie du

6. Soulignons qu'il est prévu de limiter à $7 \%$ l'augmentation des dépenses de l'État en 1984.

7. Suite au tollé provoqué par l'annonce des mesures de contrôle des changes, des correctifs ont été apportés qui ont pour effet d'amputer considérablement les bénéfices escomptés au chapitre de la réduction des sorties de devises.

8. De l'ordre de 200000 en 1983 ainsi qu'en 1984 selon les estimations de l'INSEE. 
système monétaire européen, cours du franc flottant, élévation des barrières tarifaires, politique industrielle privilégiant la constitution de filières intégrées au niveau national plutôt que de créneaux compétitifs sur le marché mondial. Les coûts et les bénéfices d'une rupture même partielle de la France avec son environnement économique sont difficiles à évaluer. Pour l'instant, ce scénario, que préconisent avec des variantes le Parti Communiste et la tendance CERES du Parti socialiste, est bel et bien écarté par le gouvernement. Mais les hésitations qui ont précédé l'adoption du second plan de rigueur indiquent bien qu'il pourrait à nouveau être tenté d'y recourir si la politique actuelle tarde à produire ses fruits.

La marge de manouvre du gouvernement apparaît donc pour le moins étroite. Au niveau du financement des régimes de sécurité sociale par exemple, il est coincé entre d'un côté une augmentation des dépenses bien évidemment supérieure à celle des recettes ${ }^{9}$, du fait du ralentissement de la croissance et des effets sociaux de la crise, et de l'aurre son engagement à ne majorer ni les charges des entreprises, dont la situation financière est assez précaire, ni la part des prélèvements obligatoires (impôts et cotisations sociales) ${ }^{10}$. Seront probablement envisagées à court terme un déplafonnement et une fiscalisation partielle des cotisations, ce qui rehausserait l'effet redistributif, en fait assez limité, selon plusieurs études, de la protection sociale. Mais on va rapidement se trouver confronté à des remises en question plus profondes de l'État-providence. La crise financière qu'il traverse en France comme dans d'autres pays débouche sur

9. Le déficit à résorber pour 1983 est de l'ordre de 14 milliards de francs.

10. Le taux de ces prélevvements (par rapport au produit intérieur brut) qui se chiffrait à environ $43 \%$ lors de l'arrivée des socialistes au pouvoir va vraisemblablement atteindre les $45 \%$ cette année. Alors que dans les pays anglo-saxons et scandinaves les impôts constituent la source la plus importante de recettes pour le trésor public, ce qui correspond à une fiscalisation totale ou partielle des systèmes de sécurité sociale, en France la part des recettes constituée par les cotisations sociales est prépondérante. 
une crise de légitimité, dont viennent témoigner de récents essais publiés tant à «droite» qu’à «gauche».

Une situation similaire se dessine au chapitre du temps de travail. Une seconde diminution obligatoire de sa durée légale n'est guère envisageable dans la conjoncture actuelle sans qu'elle ne se répercute au niveau des salaires. La création d'emplois par le partage du travail passe donc par un partage des revenus que rejerte les centrales syndicales, à l'exception de la CFDT. Les obstacles à la «révolution du temps choisi» sont considérables. Dans un contexte de faible croissance et compte tenu des répercussions du progrès technologique, on peut pourtant se demander comment il serait possible de résorber le chômage sans passer par une réduction radicale du temps de travail.

Ce bilan des deux premières années de gouvernement socialiste en France ne saurait être complet sans jeter un regard, nécessairement bref, du côté des trois grandes réformes entreprises sur les plans économique, politique et social.

\section{Les grandes réformes}

\section{a) Nationalisations}

Les nationalisations ont de tout temps occupé une place centrale dans le programme de la gauche française, constituant en quelque sorte le point d'ancrage de sa stratégie économique. Les principes traditionnellement invoqués en leur faveur sont la lutte contre les monopoles et la direction des secteurs-clés. Aux yeux de leurs promoteurs, les nationalisations constituent ainsi la base du développement de l'industrie française, sur le plan technologique notamment, et dans la conjoncture actuelle, un outil privilégié pour relancer l'économie, par le biais d'une politique d'investissements.

Une fois au pouvoir, les socialistes se sont donc fait fort de concrétiser leurs engagements en ce domaine, procédant au plus 
vaste transfert de propriété au profit de l'État jamais réalisé en Occident. Cinq grands groupes industriels (Péchiney, SaintGobain, Thomson-Brandt, Rhône Poulenc et la Compagnie générale d'électricité) ont été nationalisés à 100\%. L'État a aussi pris le contrôle en devenant actionnaire majoritaire d'autres grosses firmes telles Dassault, Matra et C.I.I. - HoneywellBull. Les banques qui appartenaient encore au secteur privé ont été nationalisées, sauf les banques étrangères, coopératives ou mutualistes, de même que les compagnies financières Suez et Paris-Bas.

Le secteur public, qui a en gros doublé de taille, fait maintenant à peu près $25 \%$ du chiffre d'affaires de l'industrie française et représente plus du tiers de l'investissement des sociétés. L'État a acquis une place prépondérante dans les industries de base (mines de fer, aciéries, chimie de base) et dans les industries à technologie avancée (armement, électronique). Près du cinquième des emplois salariés de l'industrie et du commerce se trouvent maintenant au sein du secteur nationalisé. Si l'on ajoute les personnels de l'administration, des collectivités locales et de l'ensemble des services publics, on arrive à un total de pratiquement 6 millions de salariés, soit presque un travailleur sur trois. ${ }^{11}$

Sur un autre plan, on doit souligner que les entreprises du secteur public élargi ont été dotées d'une large autonomie de gestion. Les dirigeants ${ }^{12}$, nommés par l'État, restent donc mâ̂tres des choix industriels de leurs groupes. Ceux-ci demeurent libres par exemple d'acquérir ou de céder des filiales, de licencier du personnel. Cette autonomie de gestion doit cependant s'exercer dans le cadre des contrats de plan négociés avec leur minis-

1I. Thierry Paquot, «Nationalisations, une politique conformiste», Autogestions, no 10, été 1982, 22.

12. Dans l'ensemble, peu de postes importants à la tête des sociétés nationalisées ont été confiés à des individus très proches du nouveau régime. 
tère de tutelle, contrats fixant les objectifs du développement des entreprises ainsi que les engagements réciproques entre celles-ci et l'État. Le gouvernement se réserve ainsi la possibilité d'influer sur l'orientation des firmes.

Les négociations préalables à la signature de ces contrats se sont avérées particulièrement laborieuses, notamment au chapitre de l'attribution des enveloppes financières ${ }^{13}$. L'harmonisation des stratégies particulières des groupes avec les orientations du Plan et les grands équilibres socio-économiques constitue une opération délicate. Au risque que les entreprises nationalisées s'érigent en féodalités, privilégiant leurs intérêts à court terme, s'oppose le danger d'un interventionnisme tâtillon susceptible de paralyser leur dynamisme. Il faudra encore attendre quelque temps pour voir dans quel sens s'effectuera l'aménagement de ces rapports.

Les nationalisations devaient aussi permettre de promouvoir une nouvelle politique sociale. Les entreprises publiques sont en effet conçues comme des champs d'expérimentation; on attend d'elles, selon les mots prononcés par François Mitterrand lors de la nomination des directeurs des nouvelles entreprises nationalisées, "qu'elles développent de nouvelles relations sociales et deviennent un lieu de progrès significatif pour la formation et la condition des travailleurs».

Dans cette perspective, la loi de nationalisation des grands groupes industriels et financiers a offert à leurs salariés un tiers

13. Il semble que les socialistes aient sous-estimé l'ampleur des difficultés financières des groupes industriels concernés. Ainsi, les onze entreprises nationalisées, anciennes et nouvelles, du secteur concurrentiel ont enregistré en 1981 un déficit global de 12,5 milliards de francs, leurs pertes devant s'élever à plus de 15 milliards de francs en 1982. Face à cela, l'État a injecté près de 10 milliards de francs en 1982; et 20 milliards, dont 12,5 le seront sous forme de dotation budgétaire en capital en 1983. Jean-Marie Quatrepont, "Gérer les nationalisations», Le Monde, 4 février 1983. 
des sièges dans chaque conseil d'administration ${ }^{14}$. Mais il demeure que loin d'être conçues comme la socialisation des instruments de production, les nationalisations «ne donnent lieu, pour le moment, à aucune initiative sociale originale, aucun commencement de participation ouvrière à la gestion de l'entreprise. Tout se passe comme si après avoir fait des nationalisations le signe emblématique de la transition au socialisme, on paraissait embarrassé pour s'en servir réellement ${ }^{15}$.

On peut ajouter qu'il n'est nulle part fait mention de la possibilité d'élaborer une autre politique industrielle, de profiter du contrôle étatique pour remettre en question tant les finalités que les conditions de mise en ouvre de la production. En somme, il appert que l'extension du secteur public n'a pas été l'occasion d'une rupture avec la logique capitaliste de production.

b) Décentralisation

L’État français est reconnu pour son fort centralisme et son caractère bureaucratique. En prenant le pouvoir, les socialistes se sont fixé comme priorité de corriger ce qui apparaissait comme anachronique en comparaison avec d'autres pays occidentaux en procédant à la décentralisation de l'appareil politicoadministratif. La réforme entreprise à ce chapitre a comme objectif général le transfert de pouvoirs, de compétences et de ressources de l'État central vers les élus des collectivités territoriales de différents niveaux: communes, départements et régions.

14. On retrouvera une évaluation sommaire et «à chaud» des premiers effets de cette mesure dans Marie-Claude Betbeder, «Messieurs les syndicalistes administrateurs", Le Monde, 23-24 janvier 1983. La loi de démocratisation du secteur public est venue confirmer cette orientation: dans toutes les sociétés de plus de 200 salariés dont le capital est constitué à plus de $50 \%$ par des fonds publics, les conseils d'administration ou de surveillance doivent désormais comprendre un tiers de représentants élus par l'ensemble du personnel.

15. Jacques Juilliard, «Mitterrand entre le socialisme et la république», Interventions, no. 1, nov.-déc. $1982,101$. 
Plutôt que de vouloir réaliser cette opération d'envergure d'un seul coup, ce qui aurait eu pour effet d'en retarder la mise en ouvre, ils ont préféré agir vite et créer une situation irréversible, en enclenchant aussitôt la première étape d'un processus devant s'échelonner jusqu'en 1985; celle qui correspond au transfert des pouvoirs. C'est ainsi qu'a été votée en mars 1982 la première loi de décentralisation, dite "loi sur les droits et libertés des communes, des départements et des régions», qui modifie nettement certains caractères de l'administration locale française. Sans entrer dans le détail d'une mécanique assez complexe, on peut en dégager les éléments suivants. D'abord, pour toutes les collectivités, les tutelles administratives a priori sont supprimées, c'est-à-dire que les décisions des élus locaux deviennent "exécution de plein droit», ${ }^{16}$ et les tutelles financières et techniques sont allégées. Au niveau des départements, le conseil général devient l'instance souveraine et son président élu l'organe exécutif. La région accède au statut plein et entier de collectivité territoriale - à égalité avec les communes et les départements - administrée par un conseil régional lui aussi élu au suffrage universel direct. "Le conseil régional a compétence pour promouvoir le développement économique, social, sanitaire, culturel et scientifique de la région et l'aménagement de son territoire et pour assurer la préservation de son identité, dans le respect de l'intégrité, de l'autonomie et des attributions des départements et des communes " ${ }^{17}$. Certaines tâches précises lui sont confiées telles que «concourir par ses avis à l'élaboration du plan national; élaborer et approuver le plan régional dans le respect des orientations du plan national; favoriser la coordination des investissements publics locaux dans la ré-

16. Un grand nombre de décisions à caractère local éraient en effet soumises jusqu'alors à l'autorisation du préfet, nommé par le gouvernement. Cité in «Les textesclés de la décentralisation», Aujourd'bui, no. 59, janvier-février 1983, 68.

17. Ibid. 
gion" ${ }^{18}$. Toujours au niveau de la région, on retrouve un comité économique et social qui fait office d'assemblée consultative auprès du conseil régional et de son président. Sa composition a été modifiée ${ }^{19}$ et des mesures adoptées pour en assurer un fonctionnement plus efficace. Enfin, conséquence de la reconnaissance de l'exécutif départemental et régional, le rôle du préfet, Commissaire de la République selon sa nouvelle appellation, est transformé. Il perd le contrôle des services préfectoraux qui effectuaient des tâches départementales et régionales, tandis que sa responsabilité sur les services extérieurs de l'État est renforcée.

Dans le prolongement de la loi redéfinissant les droits et libertés des collectivités locales, la Corse ainsi que les départements d'outre-mer ont été dotés de statuts particuliers, notamment d'assemblées régionales bénéficiant de pouvoirs élargis. Un texte relatif à l'organisation administrative des trois principales villes de France, Paris, Lyon et Marseille, a également été adopté. Dans un souci affirmé de démocratisation des affaires municipales, on y a mis en place des conseils d'arrondissements, élus au suffrage universel, qui se sont vus déléguer un certain nombre d'attributions en matière de gestion d'équipements collectifs et d'administration de la vie de quartier. Les associations qui exercent leurs activités sur le territoire de l'arrondissement pourront à l'occasion participer aux débats de ce conseil avec voix consultative.

Conformément à la démarche initiale, au transfert des pouvoirs doit venir se greffer une répartition des compétences entre les communes, les départements, les régions et l'État. Se-

18. Les conseils économiques et sociaux régionaux comptent désormais autant de représentants des syndicats de salariés que des employeurs, soit $35 \%$ des sièges chacun; $25 \%$ des sièges va aux organismes qui participent à la vie collective de la région et $5 \%$ à des personnalités qualifiées.

19. «Les textes-clés de la décentralisation», op. cit., p. 72. 
lon cette répartition, consignée dans un projet de loi en discussion,

«la commune se voit reconnaître la maîtrise du sol, c'est-à-dire l'essentiel des compétences dans le domaine de l'urbanisme et la responsabilité des équipements de proximité. Au département tevient la mission de solidarité et de péréquation par la gestion de services lourds et plus particulièrement l'aide sociale ainsi que par la redistribution entre les communes, notamment rurales. La région, enfin, est renforcée dans sa capacité de réflexion, d'incitation, d'impulsion dans les domaines de la planification, de l'aménagement du territoire et, plus généralement, de l'action économique et du développement " ${ }^{19}$.

Les transferts de compétences s'effectueront par étapes, chacune étant liée au vote du budget correspondant. Au total, une dizaine de domaines sont concernés: urbanisme, logement, formation professionnelle, aménagement du territoire, action sociale, santé, transports, éducation, culture, environnement.

Il est encore difficile de mesurer la portée réelle de tout ce dispositif. Dans la mesure où les élus locaux bénéficient désormais du pouvoir d'appliquer leurs politiques, on peut considérer qu'il s'agit d'un progrès dans le sens de la démocratisation. Mais la question se pose de savoir si la décentralisation se fera essentiellement au profit des notables et d'une technocratie locale, ou si elle favorisera une participation accrue des citoyens dans la «cité». Ce problème est plus ou moins laissé de côté pour l'instant. Ainsi la loi de mars 1982 demeure fidèle au modèle de la démocratie représentative; «elle ne promeut pas une démocratie participative qui rechercherait systématiquement la confrontation entre élus et forces sociales organisées ou l'intervention des citoyens ou des groupes dans l'élaboration des décisions qui les concernent ${ }^{20}$. Une loi est tout de même prévue sur la participation des citoyens à la vie locale.

20. $\quad 1$ bid, 70 . 


\section{c) Droits des travailleurs}

Les rapports sociaux dans l'entreprise sont un autre domaine pour lequel la France accusait un certain retard, notamment par rapport à ses voisins caractérisés par une politique social-démocrate. L'autoritarisme, l'arbitraire, la raideur du comportement de la hiérarchie caractérisent en effet bon nombre d'entreprises françaises. Pour tenter de remédier à cette situation, quatre lois, communément appelés lois Auroux, instituant de nouveaux droits pour les travailleurs ont été adoptées. Disons tout de suite que ces lois, dont on ne pourra mesurer l'impact véritable que dans quelques années, créent des droits à exercer, c'est-à-dire qu'elles offrent un cadre, des moyens dont les travailleurs doivent se saisir pour élargir leur place dans les entreprises.

L'aspect le plus fondamental des lois Auroux réside probablement dans la reconnaissance d'un droit d'expression direct pour les travailleurs. Ceux-ci pourront ainsi débattre entre eux, pendant le temps de travail, des conditions de travail et de son organisation, et proposer des changements. Spécifions que dans les entreprises de 200 salariés et plus les modalités d'exercice de ce droit sont à négocier avec les organisations syndicales. L'autre changement majeur consiste dans l'institution d'une négociation collective dans chaque entreprise. On doit en effet se rappeler qu'en France cette négociation s'effectue pour l'essentiel au niveau des grandes branches industrielles ou par le biais de pourparlers interprofessionnels tenus à l'échelle nationale ${ }^{21}$. Il y a donc dorénavant obligation pour les employeurs de négocier chaque année, dans les entreprises où existent une ou plusieurs sections syndicales, les salaires ainsi que la durée effective et l'organisation du temps de travail. Pour faciliter la négociation

21. En avril 1981, seulement $10 \%$ des entreprises, représentant à peine le quart des salariés, avaient un accord d'entreprise. 
de tels accords collectifs dans les petites entreprises, sont proposées la mise en place de commissions paritaires locales et la désignation de délégués interentreprises. Comme on pouvait s'y attendre, cette obligation de négocier, comme le droit d'expression, ont suscité une très vive opposition de la part du patronat qui privilégie l'expression individuelle des salariés, canalisée dans le meilleur des cas dans des cercles de qualité, groupes de progrès, ou autres expériences du même type.

Parmi les autres dispositions des lois Auroux, mentionnons le renforcement des moyens et possibilités d'interventions économiques du comité d'entreprise, la rénovation des comités d'hygiène et de sécurité - étendus à toutes les entreprises de plus de 50 salariés - dont le rôle est élargi à toutes les conditions de travail et les moyens d'action renforcés, de même d'ailleurs que ceux de la section syndicale, l'amélioration des conditions d'exercice des fonctions de délégué du personnel ou de membre du comité d'entreprise, le renforcement des moyens d'action contre les discriminations dont sont l'objet les travailleuses, enfin une limitation de l'arbitraire contenu dans les règlements intérieurs d'entreprise et la procédure disciplinaire ${ }^{22}$.

Par ce dispositif, l'intervention syndicale bénéficie très clairement d'une impulsion considérable. Cela pose toutefois deux séries de problèmes. Il y a d'abord bien entendu le risque d'un décalage grandissant entre les travailleurs, ceux des entreprises non syndiquées ayant peu de possibilités de réellement profiter des nouveaux droits. De plus, là où existent des sections syndicales, comment s'effectuera l'harmonisation entre le renforcement de leurs prérogatives et l'expression directe des travailleurs que l'on veut favoriser? C'est aux côtés d'une multiplicité d'instances collectives (comité d'entreprise, comité d'hygiène et

22. Les nouveaux droits des travailleurs Le Monde dossiers et documents. No. 102, juin 1983. "Quels sont les droits nouveaux?», Aujourd'bui, no. 58, novembredécembre 1982, pp. 15-16. 
sécurité, section syndicale, délégué du personnel) contrôlées par les syndicats que doivent prendre place les groupes d'expression directe.

Il faut aussi s'attendre à ce que les directions d'entreprise tentent de contrôler ces groupes et de les détourner au profit d'une politique visant avant tout à améliorer les performances et la productivité. Mais contrairement à tout ce qui s'apparente aux cercles de qualité, dont les participants sont triés sur le volet par l'encadrement, les nouveaux groupes d'expression réuniront de droit l'ensemble des salariés ${ }^{23}$. Malgré les écueils, une brèche est donc ouverte pour l'expérimentation de formes de démocratie à la base, vécue sur le terrain même de l'atelier de travail.

\section{Conclusion}

Si personne ne peut nier l'importance des transformations entreprises au niveau des structures économiques, de l'appareil politico-administratif et des rapports sociaux, force est pourtant de constater que le gouvernement n'a pas su impliquer la base sociale de la gauche dans son projet et appuyer ses actions de réforme sur une réelle mobilisation populaire. La priorité résidait plutôt dans la recherche d'un compromis entre les différentes forces sociales. Par ailleurs, le tournant radical dans la stratégie de sortie de crise ne s'est pas fait sans grincements de dents, tant de la part des syndicats, du partenaire communiste que de certains élus et dirigeants du Parti socialiste.

23. Danielle Rouard, «Le droit à l'expression dans l'entreprise, Le Monde, 5-6 juin 1983 , p. 17. Au $1^{\mathrm{er}}$ mai, soit trois mois après l'ouverture des négociations, des accords avaient été recensés dans $15 \%$ des 6000 entreprises concernées. Le rôle de l'encadrement se trouve généralement confirmé pour l'animation des groupes. $50 \%$ des accords prévoient qu'ils devraient se réunit 3 ou 4 fois par année, leur objet se conformant dans la quasi-totalité des cas à ce qui a été défini dans les textes ministétiels. 
Sous le poids des contraintes extérieures, l'espoir d'une alternative aux politiques conservatrices et monétaristes pratiquées ailleurs a pris un rude coup. Certes on s'en écarte encore, notamment par les mesures sociales de lutte contre le chômage (contrats de solidarité, pré-retraite, sans oublier la retraite à 60 ans, en vigueur depuis le $1^{\text {er }}$ avril dernier), l'attention accordée à la protection du pouvoir d'achat des plus bas salariés, et à la sauvegarde de la protection sociale. Mais l'expérience de la gauche en France, ainsi qu'en Grèce et en Espagne, montre bien que la marge de manœuvre demeure étroite dans la conjoncture actuelle.

Les difficultés rencontrées par le gouvernement et les désillusions qu'elles ont suscitées se sont déjà traduites par une baisse du soutien accordé à la gauche lors des élections municipales de mars dernier ${ }^{24}$ Le printemps a par la suite été l'occasion d'une forte montée de mécontentements catégoriels (commerçants, corps médical, agriculteurs, étudiants, policiers) bien sûr exploités par l'opposition mais qu'on ne saurait concevoir uniquement comme le fruit de manipulations politiques.

Les différents courants présents à l'intérieur du Parti socialiste ont aussi commencé à s'exprimer de plus en plus ouvertement à l'approche du congrès de cet automne ${ }^{25}$. Reste à voir si le gouvernement saura y puiser un second souffle. Après avoir réalisé bon nombre des engagements formulés par François Mitterrand lors des élections présidentielles, il semble maintenant à court de projet, guidé par le pragmatisme que lui inspire le rétablissement des équilibres économiques. On gouverne à vue,

24. Les divers sondages effectués depuis les municipales confirment cette chute dans la confiance accordée au Président et au gouvernement.

25. De vives critiques de la politique gouvernementale ont été formulées notamment par Jean-Pierre Chevènement, un des chefs de file du CERES et ministre de la recherche et de l'industrie avant le remaniement de mars 1983, (Le Monde, 10 et $11 \mathrm{mai}$ 1983). Pour une présentation des thèses de ce courant, on peut consulter Jacques Mandrin, Le socialisme et la France, Le Sycomore, 1983. 
prenant garde de trop heurter les divers corporatismes exacerbés en ces temps difficiles ${ }^{26}$. Parallèlement, le discours se transforme. Les chefs d'entreprise se voient réhabilités; tous les Français sont appelés à participer à l'effort national que nécessite la victoire contre le déficit extérieur et l'inflation.

L'opposition se fait quant à elle toujours plus virulente dans sa critique du gouvernement, allant jusqu’à remettre en cause plus ou moins explicitement sa légitimité. Elle semble toutefois incapable de définir une autre politique économique et sociale et demeure pour le moins évasive quant au sort qu'elle réserverait à l'héritage socialiste. Elle est néanmoins allée porter le combat sur le terrain du livre: pas moins d'une vingtaine d'ouvrages, analyses ou pamphlets, ont été publiés par les principaux ténors de la droite depuis mai 1981.

La morosité qui habite la gauche française s'est répercutée sur le plan de la production intellectuelle. Comme l'exprime Pierre Rosanvallon,

«les idéologies et les grandes synthèses macro-explicatives sont en recul. La société française semble s'être mise à l'écoute des faits. Chacun se sent plus réaliste (...) mais cet apprentissage du téalisme s'est accompagné d'une liquéfaction des représentations de l'avenir, d'un blocage de l'imagination ${ }^{27}$.

Ce désarroi idéologique, s'il est assez nettement perceptible, ne doit pas occulter l'émergence d'un certain nombre de contributions qui cherchent à dépasser le cadre de la gestion quotidienne de la crise pour tracer quelques lignes d'un au-delà différent. C'est à la société civile qu'on est tenté (les Gorz, Rosanvallon, Minc pour ne citer que ceux-là) de s'en remettre plutôt qu'à ce

26. François de Closet dans son ouvrage Toujours plus (Grasset, 1982), devenu un véritable best-seller, dresse un inventaire des divers privilèges et corporatismes qui façonnent la société française.

27. Pierre Rosanvallon, Misère de l'économie, Seuil, 1983, 149. 
monstre omnipotent, ingouvernable, niveleur qu'est l'État. Les deux tendances du socialisme français - étatiste et libertaire sont encore bien présentes, même si elle ne brûle plus autant du feu de l'utopie. Il reste à voir si de leur confrontation saura émerger un nouveau dynamisme dans le camp du changement. 